\title{
Which nutrition screening tool should be used in oncology outpatient clinics?
}

\author{
A. Donnelly ${ }^{1}$, B. Talwar ${ }^{2}$, P. Christodoulou ${ }^{2}$ and M. Y. Chan ${ }^{1}$ \\ ${ }^{1}$ Department of health and human sciences, London Metropolitan University, London N7 8DB, UK and \\ ${ }^{2}$ Dietetics department, University College London Hospital, London, UK
}

The prevalence of malnutrition in cancer patients is well documented. The consequences of this lead to poor response to treatment and increased mortality risk $^{(1)}$. There is a clear need for a Dietitian in the oncology setting ${ }^{(2)}$ as nutrition intervention has been shown to improve the nutritional status of oncology patients ${ }^{(3)}$. What is less clear is what method to use for nutritional screening especially in the outpatient setting. The Malnutrition Universal Screening Tool (MUST) has been validated for screening both inpatients and outpatients at risk of malnutrition ${ }^{(4,5)}$. MUST has shown low sensitivity and specificity in at-risk cancer patients ${ }^{(6,7)}$. The Patient-Generated Subjective Global Assessment tool (PG-SGA) has been validated for use specifically in cancer patients as an assessment tool for malnutrition risk ${ }^{(8)}$. This assessment tool is made up of five worksheets that include a physical examination of the patient. The aim of this study was to compare the use of an abridged PG-SGA and MUST tools in a chemotherapy outpatient clinic at a Regional Cancer Centre. The findings would be used to suggest a suitable malnutrition screening tool for use in oncology outpatient clinics.

Sixty-five adult patients attending the chemotherapy outpatient clinic at University College London Hospital (UCLH) were screened using the MUST and an abridged PG-SGA tool. The abridged PG-SGA used worksheets 1 and 2 of the five available worksheets. Only cancer patients who were currently seeing a dietitian were excluded from the study.

Data were collected for 2 weeks and approval for the research as a service improvement project was granted by the UCLH ethics committee. The following non-parametric tests were used to analyse the data; Wilcoxon Signed Rank test, Pearson's Correlation coefficient and Kappa test for agreement. Cross tabulations were run to examine the relationship between variables.

Of the different cancer types the highest percentage of patients were diagnosed with gynaecological cancers at $29 \%$. The tests showed a significant difference in dietetic referral between the MUST and abridged PG-SGA $(P=0.000)$. There was little correlation between the abridged PG-SGA and MUST $(r=0.325, P=0.008)$ and no agreement between the two tools $(k=0.254)$. The abridged PG-SGA identified $49 \%$ of patients for referral to the dietitian compared to $18 \%$ by MUST. The PG-SGA $(n 14)$ referred more patients with BMI's over $25 \mathrm{~kg} / \mathrm{m}^{2}$ than MUST (n 3).

In conclusion there was little agreement between the MUST and PG-SGA tools. The PG-SGA identified more patients in need of referral to a dietitian and identified more patients with BMI's greater that $25 \mathrm{~kg} / \mathrm{m}^{2}$. This is especially beneficial for Breast and Gynaecological cancer patients whose weight loss maybe masked by tumour size or ascites ${ }^{(9)}$. Future studies should look at validating the abridged tool within cancer outpatient clinics. It is recommended that the PG-SGA tool be used for screening oncology outpatients above the MUST.

1. Ottery FD (1996) Nutrition 12, S15-S19.

2. Gosselin T, Gilliard L \& Tinnen R (2008) Clin J Oncol Nurs 12(5), 781-787.

3. Isenring E, Capra S \& Bauer J (2004) Br J Cancer 91, 447-452.

4. Stratton R, Hackston A, Longmore D et al. (2004) Br J Nutr 92, 799-808.

5. British Association for Parenteral and Enteral Nutrition (BAPEN) (2003) Redditch: BAPEN

6. Huhmann M \& August D (2008) Nutr Clin Prac 23, 182-188.

7. Roulston F \& McDermot R (2009) Proc Nutr Soc 67 (OCE7), E260.

8. Bauer J, Capra S \& Ferguson M (2002) Eur J Clin Nutr 56, 779-785.

9. Laky B, Janda M, Cleghorn G et al. (2008) Am J Clin Nutr 87(6), 1678-1685. 